\title{
Metástase endotraqueal de adenocarcinoma de cólon
}

\author{
Endotraqueal metastasis of colon adenocnarcinoma \\ Rodrigo Vasconcellos Gusmão ${ }^{1}$, Samuel Bichara Melinn', Vicente Dorgan Neto ${ }^{2}$, Marcio Botter ${ }^{3}$, \\ Roberto Saad Junior ${ }^{4}$, Guilherme Carvalho ${ }^{5}$, José Luis Ferreira Filho ${ }^{5}$, Bianca Ribeiro Rodrigues ${ }^{5}$
}

\section{Resumo}

O adenocarcinoma colorretal é uma neoplasia muito frequente, originando metástases para diversos órgãos do corpo. Seus sintomas envolvem o sistema digestivo e podem variar. A doença tem uma evolução rápida, tornando o prognóstico mais reservado para casos com diagnóstico tardio. Uma disseminação metastática rara desse tipo de câncer é a região endotraqueal, apresentamos o relato de uma paciente de 46 anos, com história de adenocarcinoma colorretal T 3 N 1 M1, submetida a retossigmoidectomia, quimioterapia e radioterapia neoadjuvantes. Com evolução, em 3 anos, de metástase pulmonar tratada com segmentectomia pulmonar e lobectomia inferior direita. Após um ano da operação, ainda em quimioterapia, desenvolveu metástase endotraqueal confirmada por estudo anatomopatológico e imunohistoquímica.

Descritores: Neoplasias da traqueia, Adenocarcinoma, Neoplasias colorretais, Metástase neoplásica

\begin{abstract}
Colorectal adenocarcinoma is a very common type of neoplasia, originating metastasis for several organs of the body. The symptoms involve the digestive system and can vary. This disease has a quick evolution, making the prognosis
\end{abstract}

1. Acadêmico da Faculdade de Ciências Médicas da Santa Casa de São Paulo - $4^{\circ}$ Ano do Curso de Graduação em Medicina

2. Professor Adjunto da Faculdade de Ciências Médicas da Santa Casa de São Paulo - Departamento de Cirurgia

3. Professor Assistente da Faculdade de Ciências Médicas da Santa Casa de São Paulo-Departamento de Cirurgia

4. Professor Titular da Faculdade de Ciências Médicas da Santa Casa de São Paulo - Departamento de Cirurgia

5. Residente de Cirurgia Torácica da Irmandade da Santa Casa de Misericórdia de São Paulo - Departamento de Cirurgia

Trabalho realizado: Irmandade da Santa Casa de Misericórdia de São Paulo - Departamento de Cirurgia

Endereço para Correspondência: Rodrigo Vasconcellos Gusmão. Rua Raul Vicente, 202, Casa 04 -02370-010 - São Paulo - SP Brasil. Tel.: (11) 97610-9971. more reserved for cases with late diagnosis. A very rare type of metastatic dissemination of this type of cancer is the endotracheal region, we present the case of a 46-year-old patient, with history of colorectal adenocarcinoma $T$ 3 N 1 M1, subjected to rectosigmoidectomy and neoadjuvant chemotherapy and radiotherapy. With evolution, in 3 years, of pulmonary metastasis treated with pulmonary segmentectomy and a lower right lobectomy. After a year of surgery, still in chemotherapy, developed endotracheal metastasis confirmed by anatomopathological study Immunohistochemical study.

Keywords: Tracheal neoplasms, Adenocarcinoma, Colorectal neoplasms, Neoplasms metastasis

\section{Introdução}

Adenocarcinoma colorretal é a terceira neoplasia mais comum no mundo. Aproximadamente 20\% dos pacientes apresentam metástases no momento do diagnóstico e seu desenvolvimento piora consideravelmente o prognóstico ${ }^{(1)}$. Os principais locais afetados são fígado, pulmões, peritônio e linfonodos à distância ${ }^{(2)}$.

Os pulmões são frequente área de surgimento metastático, no entanto neoplasias de origem não pulmorar em regiões endotraqueais e endobrônquicas são muito raras, representando apenas $5 \%$ dos casos na árvore pulmonar ${ }^{(3-4)}$. Além disso, a condição é tão incomum que se mostra, apenas, como 1,5\% dos casos de metástase de adenocarcinoma colorretal ${ }^{(2)}$.

Os principais locais de origem das neoplasias com esse tipo de metástase relatados na literatura foram tumores mamários, carcinoma de células renais, melanoma e carcinoma colorretal ${ }^{(3,5)}$. Assim como carcinoma de tireoide, testículo, pênis, nervo olfatório, próstata, bexiga, ovário, colo uterino, pâncreas, esôfago, estomago e sarcoma ${ }^{(5)}$.

A ocorrência de metástases endotraqueais de tumores de cólon é acompanhada de disseminação metastática sincrônica, sendo, portanto, sinal de doença avançada e de mau prognóstico, com sobrevivência curta. O tempo médio para o diagnóstico dessas neo- 
plasias endotraqueais/endobrônquicas,a partir da identificação do adenocarcinoma colorretal, é de 43 meses, e a sobrevivência média, após esse intervalo, é de 15 meses $^{(5)}$.

\section{Relato de Caso}

Paciente de 46 anos, com história de adenocarcinoma de reto (T3 N1 M1), tratado com retossigmoidectomia há 3 anos, quimioterapia neoadjuvante e radioterapia. Posteriormente, foi submetida a segmentectomia pulmonar e lobectomia inferior direita por metástases pulmonares. Um ano após as operações, ainda em vigência de quimioterapia, apresentava tomografia de tórax (figura 1) e broncoscopia (figura 2) com lesão intraluminal polipoide da traqueia, Em terço proximal. Realizada broncoscopia, na qual foi constatada lesão pediculada em parede anterior direita, ocluindo $70 \%$ da luz. Optado por ressecção endoscópica da lesão.

Após análise anatomopatológica e imunohistoquímica, confirmou se tratar de metástase de adenocarcinoma de reto.

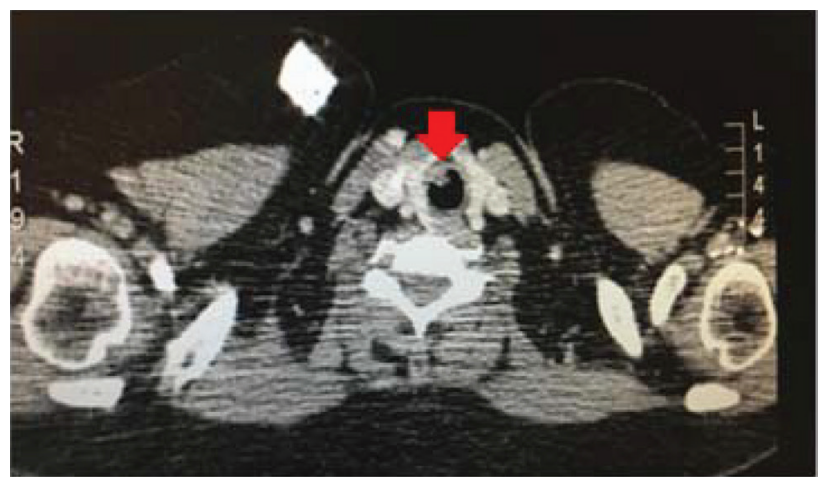

Figura 1 - Tomografia Computadorizada de tórax com lesão intraluminal na traqueia.

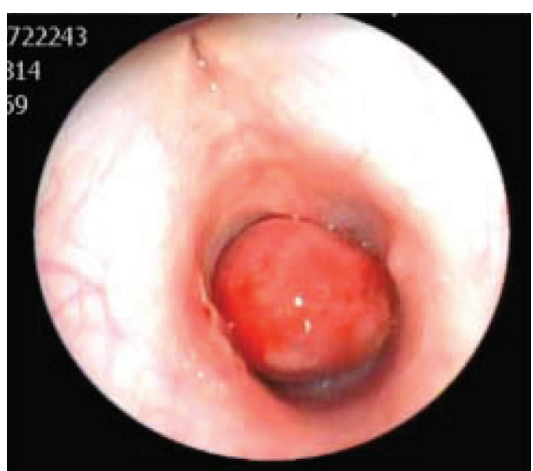

Figura 2 - Broncoscopia com lesão polipoide intraluminal.

\section{Conclusão}

Trata-se de um caso no qual a paciente apresenta uma metástase endotraqueal de adenocarcinoma de cólon. Este tipo de disseminação é de natureza rara e apresenta baixo tempo de sobrevivência.

Dado a raridade e o mal prognostico de pacientes com esse tipo de metástase, o estudo dos poucos casos relatados na literatura permite maior conhecimento da doença para melhor manejo dos pacientes.

\section{Referências}

1. Aakif M, Balfe P, Elfaedy O, Awan FN, Pretorius F, Silvio L, et al. Study on colorectal cancer presentation, treatment and follow-up. Int J Colorectal Dis. 2016; 31(7):1361-3.

2. Sugarbaker PH. Colorectal cancer: prevention and management of metastatic disease. Biomed Res Int. 2014; 2014:782890.

3. Serbanescu GL, Anghel RM. Can endobronchial or endotracheal metastases appear from rectal adenocarcinoma? J Med Life. 2017; 10(1):66-9.

4. Shim HK, Kwon HW, Kim TS, Kim SK. Endotracheal Metastasis Seen on FDG PET/CT in a Patient with Previous Colorectal Cancer. Nucl Med Mol Imaging. 2010; 44(4):294-6.

5. Sørensen JB. Endobronchial metastases from extrapulmonary solid tumors. Acta Oncol. 2004; 43(1):73-9.

Trabalho recebido: 02/12/2017

Trabalho aprovado: 02/08/2018

Trabalho publlicado: $10 / 12 / 2018$ 